\title{
On Observability of Fuzzy Dynamical Matrix Lyapunov Systems
}

\author{
Madhunapantula Suryanarayana Murty and Grande Suresh Kumar \\ Department of Applied Mathematics Acharya Nagarjuna University-Nuzvid Cam- \\ pus, Nuzvid, Andra Pradesh, India \\ e-mail : drmsn2002@gmail.com and gsk006@yahoo.com
}

ABStract. In this paper we generate a fuzzy dynamical matrix Lyapunov system and obtain a sufficient condition for the observability of this system.

\section{Introduction}

The importance of control theory in Applied mathematics and its occurrence in several problems such as mechanics, electromagnetic theory, thermodynamics, artificial satellites etc., are well known. The observability condition assures the construction of the state from the output. This property is intrensic for systems and play an important role in the theory of linear systems.

The objective of this paper is to provide sufficient condition for observability of first order matrix Lyapunov system described by

$$
\begin{gathered}
X^{\prime}(t)=A(t) X(t)+X(t) B(t)+F(t) U(t), \quad X(0)=X_{0}, \quad t>0, \\
Y(t)=C(t) X(t)+D(t) U(t)
\end{gathered}
$$

where $U(t)$ is a $n \times n$ fuzzy input matrix called fuzzy control and $Y(t)$ is a $n \times n$ fuzzy output matrix. Here $A(t), B(t), F(t), C(t)$, and $D(t)$ are matrices of order $n \times n$, whose elements are continuous functions of $\mathrm{t}$ on $J=[0, T] \subset R(T>0)$.

The problem of controllability and observability for systems of ordinary differential equations has been studied by Barnett [2] and for matrix Lyapunov systems by Murty, Rao and Suresh Kumar [8]. Recently the observability criteria for fuzzy dynamical control systems was studied by Ding and Kandel [5].

In section 2 we present some basic definitions and results relating to fuzzy sets and also some properties of Kronecker products and obtain general solution of the system (1.1), when $U(t)$ is a crisp continuous matrix.

In section 3 we generate a fuzzy dynamical matrix Lyapunov system and also obtain its solution set.

Received September 7, 2007.

2000 Mathematics Subject Classification: 26E50, 34A10, 49K15, 93C15.

Key words and phrases: fuzzy matrix Lyapunov system, Kronecker product, fundamental matrix, observability. 
In section 4 we introduce the notion of likely observability and present sufficient conditions for the fuzzy dynamical matrix Lyapunov system to be likely observable, and illustrate the main theorem with a suitable example.

This paper extend some of the results of Ding and Kandel [5] to fuzzy dynamical matrix Lyapunov systems.

\section{Preliminaries}

Let $P_{k}\left(R^{n}\right)$ denotes the family of all nonempty compact convex subsets of $R^{n}$. Define the addition and scalar multiplication in $P_{k}\left(R^{n}\right)$ as usual.

Radstrom [12] states that $P_{k}\left(R^{n}\right)$ is a commutative semi-group under addition, which satisfies the cancellation law. Moreover, if $\alpha, \beta \in R$ and $A, B \in P_{k}\left(R^{n}\right)$, then

$$
\alpha(A+B)=\alpha A+\alpha B, \quad \alpha(\beta A)=(\alpha \beta) A, \quad 1 . A=A
$$

and if $\alpha, \beta \geq 0$, then $(\alpha+\beta) A=\alpha A+\beta A$. The distance between $A$ and $B$ is defined by the Hausdorff metric

$$
d(A, B)=\inf \{\epsilon: A \subset N(B, \epsilon), B \subset N(A, \epsilon)\},
$$

where

$$
N(A, \epsilon)=\left\{x \in R^{n}:\|x-y\|<\epsilon, \text { for some } y \in A\right\} .
$$

Definition 2.1. A set valued function $F: J \rightarrow P_{k}\left(R^{n}\right)$ is said to be measurable, if it satisfies any one of the following equivalent conditions;

1. for all $u \in R^{n}, t \rightarrow d_{F(t)}(u)=\inf _{v \in F(t)}\|u-v\|$ is measurable,

2. $\operatorname{Gr} F=\left\{(t, u) \in J \times R^{n}: u \in F(t)\right\} \in \Sigma \times \beta\left(R^{n}\right)$, where $\Sigma, \beta\left(R^{n}\right)$ are Borel $\sigma$-field of $J$ and $R^{n}$ respectively (Graph measurability),

3. there exists a sequence $\left\{f_{n}(.)\right\}_{n \geq 1}$ of measurable functions such that $F(t)=$ $\overline{\left\{f_{n}(.)\right\}_{n \geq 1}}$, for all $t \in J$ (Castaing's representation).

We denote by $S_{F}^{1}$ the set of all selections of $F($.) that belong to the Lebesgue Bochner space $L_{R^{n}}^{1}(J)$. i.e.,

$$
S_{F}^{1}=\left\{f(.) \in L_{R^{n}}^{1}(J): f(t) \in F(t) \text { a.e. }\right\} .
$$

We present the Aumann's integral as follows;

$$
\text { (A) } \int_{J} F(t) d t=\left\{\int_{J} f(t) d t, f(.) \in S_{F}^{1}\right\} .
$$

We say that $F: J \rightarrow P_{k}\left(R^{n}\right)$ is integrably bounded if it is measurable and there exists a function $h: J \rightarrow R, h \in L_{R^{n}}^{1}(J)$ such that $\|u\| \leq h(t)$, for all $u \in F(t)$.

From [1], we know that if $F$ is closed valued measurable multifunction, then 
$\int_{J} F(t) d t$ is convex in $R^{n}$. Furthermore, if $F$ is integrably bounded then $\int_{J} F(t) d t$ is compact in $R^{n}$.

Let

$$
E^{n}=\left\{u: R^{n} \rightarrow[0,1] / u \text { satisfies }(i)-(i v) \text { below }\right\},
$$

where

(i) $u$ is normal, i.e., there exists an $x_{0} \in R^{n}$ such that $u\left(x_{0}\right)=1$;

(ii) $u$ is fuzzy convex, i.e., for $x, y \in R^{n}$ and $0 \leq \lambda \leq 1$,

$$
u(\lambda x+(1-\lambda) y) \geq \min [u(x), u(y)] ;
$$

(iii) $\mathrm{u}$ is upper semi-continuous;

(iv) $[u]^{0}=\left\{x \in R^{n} / u(x)>0\right\}$ is compact.

For $0<\alpha \leq 1$, the $\alpha$-level set is denoted and defined by

$[u]^{\alpha}=\left\{x \in R^{n} / u(x) \geq \alpha\right\}$. Then from (i)-(iv) it follows that $[u]^{\alpha} \in P_{k}\left(R^{n}\right)$ for all $0 \leq \alpha \leq 1$.

Define $D: E^{n} \times E^{n} \rightarrow[0, \infty)$ by the equation

$$
D(u, v)=\sup \left\{d\left([u]^{\alpha},[v]^{\alpha}\right) / \alpha \in[0,1]\right\},
$$

where $d$ is the Hausdorff metric defined in $P_{k}\left(R^{n}\right)$. It is easy to show that $D$ is a metric in $E^{n}$ and using results of [[4], [12]], we see that $\left(E^{n}, D\right)$ is a complete metric space, but not locally compact. Moreover the distance $D$ verifies that

$$
\begin{aligned}
& D(u+w, v+w)=D(u, v), \quad u, v, w \in E^{n}, \\
& D(\lambda u, \lambda v)=|\lambda| D(u, v), \quad u, v \in E^{n}, \lambda \in R, \\
& D(u+w, v+z) \leq D(u, v)+D(w, z), \quad u, v, w, z \in E^{n} .
\end{aligned}
$$

We note that $\left(E^{n}, D\right)$ is not a vector space. But it can be imbedded isomorphically as a cone in a Banach space [12].

Regarding fundamentals of differentiability and integrability of fuzzy functions we refer to O.Kaleva [6], Lakshmikantham and Mohapatra [7].

In the sequel, we need the following representation theorem.

Theorem 2.1([10]). If $u \in E^{n}$, then

(1) $[u]^{\alpha} \in P_{k}\left(R^{n}\right)$, for all $0 \leq \alpha \leq 1$.

(2) $[u]^{\alpha_{2}} \subset[u]^{\alpha_{1}}$, for all $0 \leq \alpha_{1} \leq \alpha_{2} \leq 1$.

(3) If $\left\{\alpha_{k}\right\}$ is a nondecreasing sequence converging to $\alpha>0$, then $[u]^{\alpha}=\bigcap_{k \geq 1}[u]^{\alpha_{k}}$.

Conversely, if $\left\{A^{\alpha}: 0 \leq \alpha \leq 1\right\}$ is a family of subsets of $R^{n}$ satisfying (1)-(3), then there exists a $u \in E^{n}$ such that $[u]^{\alpha}=A^{\alpha}$ for $0<\alpha \leq 1$ and $[u]^{0}=\bigcup_{0<\alpha<1} A^{\alpha} \subset A^{0}$.

A fuzzy set valued mapping $F: J \rightarrow E^{n}$ is called fuzzy integrably bounded if $F_{0}(t)$ is integrably bounded. 
Definition 2.2. Let $F: J \rightarrow E^{n}$ be a fuzzy integrably bounded mapping. The fuzzy integral of $F$ over $J$ denoted by $\int_{J} F(t) d t$, is defined levelsetwise by

$$
\left.\left[\int_{J} F(t) d t\right]\right]^{\alpha}=(A) \int_{J} F_{\alpha}(t) d t, 0<\alpha \leq 1 .
$$

Let $F: J \times E^{n} \rightarrow E^{n}$, consider the fuzzy initial value problem

$$
u^{\prime}=F(t, u), \quad u(0)=u_{0} .
$$

Definition 2.3. A mapping $u: J \rightarrow E^{n}$ is a fuzzy weak solution to (2.1), if it is continuous and satisfies the integral equation

$$
u(t)=u_{0}+\int_{0}^{t} F(s, u(s)) d s, \quad \forall t \in J .
$$

If $F$ is continuous, then this weak solution also satisfies (2.1) [11, Lemma 6.1] and we call it a fuzzy strong solution to (2.1).

Now we present some properties and rules for Kronecker products and basic results related to matrix Lyapunov systems.

Definition 2.4([8]). Let $A \in C^{m \times n}$ and $B \in C^{p \times q}$ then the Kronecker product of $A$ and $B$ written $A \otimes B$ is defined to be the partitioned matrix

$$
A \otimes B=\left[\begin{array}{cccccc}
a_{11} B & a_{12} B & \cdot & \cdot & . & a_{1 n} B \\
a_{21} B & a_{22} B & \cdot & \cdot & \cdot & a_{2 n} B \\
\cdot & \cdot & \cdot & \cdot & \cdot & \cdot \\
a_{m 1} B & a_{m 2} B & \cdot & \cdot & \cdot & a_{m n} B
\end{array}\right]
$$

is an $m p \times n q$ matrix and is in $C^{m p \times n q}$.

Definition 2.5([8]). Let $A=\left[a_{i j}\right] \in C^{m \times n}$, we denote

$$
\hat{A}=\operatorname{Vec} A=\left[\begin{array}{c}
A_{.1} \\
A_{.2} \\
\cdot \\
\cdot \\
A_{. n}
\end{array}\right] \text {, where } A_{. j}=\left[\begin{array}{c}
a_{1 j} \\
a_{2 j} \\
\cdot \\
\cdot \\
a_{m j}
\end{array}\right](1 \leq j \leq n) .
$$

The Kronecker product has the following properties and rules [8].

1. $(A \otimes B)^{*}=A^{*} \otimes B^{*}\left(A^{*}\right.$ denotes transpose of $\left.A\right)$

2. $(A \otimes B)^{-1}=A^{-1} \otimes B^{-1}$

3. The mixed product rule

$(A \otimes B)(C \otimes D)=(A C \otimes B D)$

this rule holds, provided the dimension of the matrices are such that the various expressions exist. 
4. $\|A \otimes B\|=\|A\|\|B\|\left(\|A\|=\sup \left\{\left|a_{i j}(t)\right|, t \in J\right\}\right)$

5. If $A(t)$ and $B(t)$ are matrices, then

$(A \otimes B)^{\prime}=A^{\prime} \otimes B+A \otimes B^{\prime}\left({ }^{\prime}=d / d t\right)$

6. Vec $(A Y B)=\left(B^{*} \otimes A\right) \operatorname{Vec} Y$

7. If $A$ and $B$ are matrices both of order $n \times n$ then

(i) $\operatorname{Vec}(A X)=\left(I_{n} \otimes A\right) \operatorname{Vec} X$

(ii) $\operatorname{Vec}(X A)=\left(A^{*} \otimes I_{n}\right) \operatorname{Vec} X$.

Now by applying the Vec operator to the matrix Lyapunov system (1.1) satisfying (1.2) and using the above properties, we have

$$
\begin{gathered}
\hat{X}^{\prime}(t)=G(t) \hat{X}(t)+\left(I_{n} \otimes F(t)\right) \hat{U}(t), \quad \hat{X}(0)=\hat{X}_{0}, \\
\hat{Y}(t)=\left(I_{n} \otimes C(t)\right) \hat{X}(t)+\left(I_{n} \otimes D(t)\right) \hat{U}(t),
\end{gathered}
$$

where $G(t)=\left(B^{*} \otimes I_{n}\right)+\left(I_{n} \otimes A\right)$ is a $n^{2} \times n^{2}$ matrix and $\hat{X}=\operatorname{Vec} X(t)$, $\hat{U}=\operatorname{Vec} U(t), \hat{Y}=\operatorname{Vec} Y(t)$ are column matrices of order $n^{2}$.

The corresponding linear homogeneous system of (2.2) is

$$
\hat{X}^{\prime}(t)=G(t) \hat{X}(t), \quad \hat{X}(0)=\hat{X}_{0} .
$$

Lemma 2.1. Let $\phi(t)$ and $\psi(t)$ be the fundamental matrices for the systems

$$
X^{\prime}(t)=A(t) X(t), \quad X(0)=I_{n},
$$

and

$$
\left[X^{*}(t)\right]^{\prime}=B^{*}(t) X^{*}(t), \quad X(0)=I_{n}
$$

respectively. Then the matrix $\psi(t) \otimes \phi(t)$ is a fundamental matrix of (2.4) and the solution of $(2.4)$ is $\hat{X}(t)=(\psi(t) \otimes \phi(t)) \hat{X}_{0}$.

Proof. Consider

$$
\begin{aligned}
(\psi(t) \otimes \phi(t))^{\prime} & =\left(\psi(t)^{\prime} \otimes \phi(t)\right)+\left(\psi(t) \otimes \phi^{\prime}(t)\right) \\
& =\left(B^{*}(t) \psi(t) \otimes \phi(t)\right)+(\psi(t) \otimes A(t) \phi(t)) \\
& =\left(B^{*}(t) \otimes I_{n}\right)(\psi(t) \otimes \phi(t))+\left(I_{n} \otimes A(t)\right)(\psi(t) \otimes \phi(t)) \\
& =\left[B^{*}(t) \otimes I_{n}+I_{n} \otimes A(t)\right](\psi(t) \otimes \phi(t)) \\
& =G(t)(\psi(t) \otimes \phi(t)) .
\end{aligned}
$$

Also $\psi(0) \otimes \phi(0)=I_{n} \otimes I_{n}=I_{n^{2}}$.

Hence $\psi(t) \otimes \phi(t)$ is a fundamental matrix of (2.4). Clearly $\hat{X}(t)=(\psi(t) \otimes \phi(t)) \hat{X}_{0}$ is a solution of (2.4). 
Theorem 2.2. Let $\phi(t)$ and $\psi(t)$ be the fundamental matrices for the systems (2.5) and (2.6), then the unique solution of (2.2) is

$$
\hat{X}(t)=(\psi(t) \otimes \phi(t)) \hat{X}_{0}+\int_{0}^{t}(\psi(t-s) \otimes \phi(t-s))\left(I_{n} \otimes \hat{F}(s)\right) \hat{U}(s) d s .
$$

Proof. First, we show that the solution of (2.2) is of the form $\hat{X}(t)=(\psi(t) \otimes$ $\phi(t)) \hat{X}_{0}+\widetilde{X}(t)$, where $\widetilde{X}(t)$ is a particular solution of $(2.2)$ and is given by

$$
\widetilde{X}(t)=\int_{0}^{t}(\psi(t-s) \otimes \phi(t-s))\left(I_{n} \otimes \hat{F}(s)\right) \hat{U}(s) d s .
$$

Let $u(t)$ be any other solution of $(2.2)$, write $w(t)=u(t)-\widetilde{X}(t)$, then $w$ satisfies (2.4), hence $w=(\psi(t) \otimes \phi(t)) \hat{X}_{0}, u(t)=(\psi(t) \otimes \phi(t)) \hat{X}_{0}+\widetilde{X}(t)$.

Consider the vector $\widetilde{X}(t)=(\psi(t) \otimes \phi(t)) v(t)$, where $v(t)$ is an arbitrary vector to be determined, so as to satisfy equation $(2.2)$.

$$
\begin{aligned}
& \widetilde{X}^{\prime}(t)=(\psi(t) \otimes \phi(t))^{\prime} v(t)+(\psi(t) \otimes \phi(t)) v^{\prime}(t) \\
\Rightarrow & G(t) \widetilde{X}(t)+\left(I_{n} \otimes \hat{F}(t)\right) \hat{U}(t)=G(t)(\psi(t) \otimes \phi(t)) v(t) \\
\Rightarrow & (\psi(t) \otimes \phi(t)) v^{\prime}(t)=\left(I_{n} \otimes \hat{F}(t) \hat{U}(t) \quad+(\psi(t) \otimes \phi(t)) v^{\prime}(t)\right. \\
\Rightarrow & v^{\prime}(t)=\left(\psi^{-1}(t) \otimes \phi^{-1}(t)\right)\left(I_{n} \otimes \hat{F}(t)\right) \hat{U}(t) \\
\Rightarrow & v(t)=\int_{0}^{t}\left(\psi^{-1}(s) \otimes \phi^{-1}(s)\right)\left(I_{n} \otimes \hat{F}(s)\right) \hat{U}(s) d s .
\end{aligned}
$$

Hence the desired expression follows immediately by noting the fact that $\phi(t) \phi^{-1}(s)=$ $\phi(t-s)$ and $\psi(t) \psi^{-1}(s)=\psi(t-s)$.

\section{Formation of fuzzy dynamical Lyapunov systems}

In this section we show that the following system

$$
\begin{gathered}
\hat{X}^{\prime}(t)=G(t) \hat{X}(t)+\left(I_{n} \otimes F(t)\right) \hat{U}(t), \hat{X}(0)=\hat{X}_{0} \\
\hat{Y}(t)=\left(I_{n} \otimes C(t)\right) \hat{X}(t)+\left(I_{n} \otimes D(t)\right) \hat{U}(t),
\end{gathered}
$$

determines a fuzzy system, when $\hat{U}(t)$ is a fuzzy set.

Fix $0<\alpha \leq 1$, let $[\hat{U}(t)]^{\alpha}$ be the $\alpha$-level set of $\hat{U}(t)$. For any positive number $T$, consider the differential inclusions

$$
\hat{X}^{\prime}(t) \in G(t) \hat{X}(t)+\left(I_{n} \otimes F(t)\right)[\hat{U}(t)]^{\alpha}, \quad t \in[0, T]
$$

$$
\hat{X}(0)=\hat{X}_{0}
$$


Let $\hat{X}^{\alpha}$ be the solution set of inclusions (3.3) and (3.4).

Claim (i). $[\hat{X}(t)]^{\alpha} \in P_{k}\left(R^{n^{2}}\right)$, for every $0 \leq \alpha \leq 1, t \in[0, T]$.

First, we prove that $\hat{X}^{\alpha}$ is nonempty, compact and convex in $C\left[[0, T], R^{n^{2}}\right]$. Since $[\hat{U}(t)]^{\alpha}$ has measurable selection, we have $\hat{X}^{\alpha}$ is nonempty. Let $K=$ $\max _{t \in[0, T]}\|\phi(t)\|, \quad L=\max _{t \in[0, T]}\|\psi(t)\|, \quad M=\max _{t \in[0, T]}\left\{\|u(t)\|: u(t) \in[\hat{U}(t)]^{\alpha}\right\}$, and $N=\max _{t \in[0, T]}\|F(t)\|$.

If for any $\hat{X} \in \hat{X}^{\alpha}$, then there is a selection $u(t) \in[\hat{U}(t)]^{\alpha}$ such that

$$
\hat{X}(t)=(\psi(t) \otimes \phi(t)) \hat{X}_{0}+\int_{0}^{t}(\psi(t-s) \otimes \phi(t-s))\left(I_{n} \otimes F(s)\right) u(s) d s .
$$

Then

$$
\begin{aligned}
\|\hat{X}(t)\| & \leq\left\|(\psi(t) \otimes \phi(t)) \hat{X}_{0}\right\|+\int_{0}^{t}\left\|(\psi(t-s) \otimes \phi(t-s))\left(I_{n} \otimes F(s)\right) u(s)\right\| d s \\
& \leq\|\psi(t)\|\|\phi(t)\|\left\|\hat{X}_{0}\right\|+\int_{0}^{t}\|\psi(t-s)\|\|\phi(t-s)\|\|F(s)\|\|u(s)\| d s \\
& \leq K L\left\|\hat{X}_{0}\right\|+K L N M T .
\end{aligned}
$$

Thus $\hat{X}^{\alpha}$ is bounded.

For any $t_{1}, t_{2} \in[0, T]$

$$
\begin{aligned}
& \hat{X}\left(t_{1}\right)-\hat{X}\left(t_{2}\right) \\
& =\left(\psi\left(t_{1}\right) \otimes \phi\left(t_{1}\right)\right) \hat{X}_{0}+\int_{0}^{t_{1}}\left(\psi\left(t_{1}-s\right) \otimes \phi\left(t_{1}-s\right)\right)\left(I_{n} \otimes F(s)\right) u(s) d s \\
& \quad-\left(\psi\left(t_{2}\right) \otimes \phi\left(t_{2}\right)\right) \hat{X}_{0}-\int_{0}^{t_{2}}\left(\psi\left(t_{2}-s\right) \otimes \phi\left(t_{2}-s\right)\right)\left(I_{n} \otimes F(s)\right) u(s) d s
\end{aligned}
$$

Therefore

$$
\begin{aligned}
\left\|\hat{X}\left(t_{1}\right)-\hat{X}\left(t_{2}\right)\right\| \leq & \left\|\left(\psi\left(t_{1}\right) \otimes \phi\left(t_{1}\right)\right)-\left(\psi\left(t_{2}\right) \otimes \phi\left(t_{2}\right)\right)\right\|\left\|\hat{X}_{0}\right\| \\
& +\int_{t_{2}}^{t_{1}}\left\|\left(\psi\left(t_{1}-s\right) \otimes \phi\left(t_{1}-s\right)\right)\left(I_{n} \otimes F(s)\right) u(s)\right\| d s \\
& +\int_{0}^{t_{2}} \|\left[\left(\psi\left(t_{1}-s\right) \otimes \phi\left(t_{1}-s\right)\right)-\left(\psi\left(t_{2}-s\right) \otimes \phi\left(t_{2}-s\right)\right)\right] \\
\leq & \left(I_{n} \otimes F(s)\right) u(s) \| d s \\
& +M N \int_{0}^{T}\left\|\left(\psi\left(t_{1}\right) \otimes \phi\left(t_{1}\right)\right)-\left(\psi\left(t_{2}\right) \otimes \phi\left(t_{2}\right)\right)\right\|\left\|\hat{X}_{0}\right\|+K L N M\left|t_{1}-t_{2}\right|
\end{aligned}
$$


Since $\phi(t)$ and $\psi(t)$ are both uniformly continuous on $[0, T], \hat{X}$ is equi-continuous. Thus $\hat{X}^{\alpha}$ is relatively compact. If $\hat{X}^{\alpha}$ is closed, then it is compact.

Let $\hat{X}_{k} \in \hat{X}^{\alpha}$ and $\hat{X}_{k} \rightarrow \hat{X}$. For each $\hat{X}_{k}$, there is a $u_{k} \in[\hat{U}(t)]^{\alpha}$ such that

$$
\hat{X}_{k}(t)=(\psi(t) \otimes \phi(t)) \hat{X}_{0}+\int_{0}^{t}(\psi(t-s) \otimes \phi(t-s))\left(I_{n} \otimes F(s)\right) u_{k}(s) d s .
$$

Since $u_{k} \in[\hat{U}(t)]^{\alpha}$, it is closed, then there exists a subsequence $\left\{u_{k_{j}}\right\}$ of $\left\{u_{k}\right\}$ converging weakly to $u \in[\hat{U}(t)]^{\alpha}$. From Mazur's theorem [3], there exists a sequence of numbers $\lambda_{j}>0, \sum_{j} \lambda_{j}=1$ such that $\sum_{j} \lambda_{j} u_{k_{j}}$ converges strongly to $u$.

Thus from (3.5), we have

$$
\begin{aligned}
\sum_{j} \lambda_{j} \hat{X}_{k_{j}}(t) & =\sum_{j} \lambda_{j}(\psi(t) \otimes \phi(t)) \hat{X}_{0} \\
& +\int_{0}^{t}(\psi(t-s) \otimes \phi(t-s))\left(I_{n} \otimes F(s)\right) \sum_{j} \lambda_{j} u_{k_{j}}(s) d s .
\end{aligned}
$$

From Fatou's lemma, taking the limit on both sides of (3.6), we have

$$
\hat{X}(t)=(\psi(t) \otimes \phi(t)) \hat{X}_{0}+\int_{0}^{t}(\psi(t-s) \otimes \phi(t-s))\left(I_{n} \otimes F(s)\right) u(s) d s .
$$

Thus $\hat{X}(t) \in \hat{X}^{\alpha}$, and hence $\hat{X}^{\alpha}$ is closed.

Let $\hat{X}_{1}, \hat{X}_{2} \in \hat{X}^{\alpha}$, then there exist $u_{1}, u_{2} \in[\hat{U}(t)]^{\alpha}$ such that

$$
\hat{X}_{1}^{\prime}(t)=G(t) \hat{X}_{1}(t)+\left(I_{n} \otimes F(t)\right) u_{1}(t)
$$

and

$$
\hat{X}_{2}^{\prime}(t)=G(t) \hat{X}_{2}(t)+\left(I_{n} \otimes F(t)\right) u_{2}(t) .
$$

Let $\hat{X}=\lambda \hat{X}_{1}(t)+(1-\lambda) \hat{X}_{2}(t), 0 \leq \lambda \leq 1$, then

$$
\begin{aligned}
& \hat{X}^{\prime}=\lambda \hat{X}_{1}^{\prime}(t)+(1-\lambda) \hat{X}_{2}^{\prime}(t) \\
& =\lambda\left(G(t) \hat{X}_{1}(t)+\left(I_{n} \otimes F(t)\right) u_{1}(t)\right)+(1-\lambda)\left(G(t) \hat{X}_{2}(t)+\left(I_{n} \otimes F(t)\right) u_{2}(t)\right) \\
& =G(t)\left[\lambda \hat{X}_{1}(t)+(1-\lambda) \hat{X}_{2}(t)\right]+\left(I_{n} \otimes F(t)\right)\left[\lambda u_{1}(t)+(1-\lambda) u_{2}(t)\right] .
\end{aligned}
$$

Since $[\hat{U}(t)]^{\alpha}$ is convex, $\lambda u_{1}(t)+(1-\lambda) u_{2}(t) \in[\hat{U}(t)]^{\alpha}$, we have

$$
\hat{X}^{\prime}(t) \in G(t) \hat{X}(t)+\left(I_{n} \otimes F(t)\right)[\hat{U}(t)]^{\alpha} .
$$

i.e., $\hat{X} \in \hat{X}^{\alpha}$. Thus $\hat{X}^{\alpha}$ is convex.

From Arzela-Ascoli theorem, we know that $[\hat{X}(t)]^{\alpha}$ is compact in $R^{n^{2}}$, for every $t \in[0, T]$. Also it is obvious that $[\hat{X}(t)]^{\alpha}$ is convex in $R^{n^{2}}$. Thus we have $[\hat{X}(t)]^{\alpha} \in$ $P_{k}\left(R^{n^{2}}\right)$, for every $t \in[0, T]$. Hence the claim. 
Claim (ii). $[\hat{X}(t)]^{\alpha_{2}} \subset[\hat{X}(t)]^{\alpha_{1}}$, for all $0 \leq \alpha_{1} \leq \alpha_{2} \leq 1$.

Let $0 \leq \alpha_{1} \leq \alpha_{2} \leq 1$. Since $[\hat{U}(t)]^{\alpha_{2}} \subset[\hat{U}(t)]^{\alpha_{1}}$, we have $S_{[\hat{U}(t)]^{\alpha_{2}}}^{1} \subset S_{[\hat{U}(t)]^{\alpha_{1}}}^{1}$ and the following inclusion

$$
\begin{aligned}
\hat{X}^{\prime}(t) & \in G(t) \hat{X}(t)+\left(I_{n} \otimes F(t)\right)[\hat{U}(t)]^{\alpha_{2}} \\
& \subset G(t) \hat{X}(t)+\left(I_{n} \otimes F(t)\right)[\hat{U}(t)]^{\alpha_{1}} .
\end{aligned}
$$

Consider the differential inclusions

$$
\hat{X}^{\prime}(t) \in G(t) \hat{X}+\left(I_{n} \otimes F(t)\right) \hat{U}^{\alpha_{2}}(t), \quad t \in[0, T]
$$

and

$$
\hat{X}^{\prime}(t) \in G(t) \hat{X}+\left(I_{n} \otimes F(t)\right) \hat{U}^{\alpha_{1}}(t), \quad t \in[0, T] .
$$

Let $\hat{X}^{\alpha_{2}}$ and $\hat{X}^{\alpha_{1}}$ be the solution sets of (3.8) and (3.9) respectively. Clearly, the solution of (3.8) satisfies the following inclusion

$$
\begin{aligned}
\hat{X}(t) & \in(\psi(t) \otimes \phi(t)) \hat{X}_{0}+\int_{0}^{t}(\psi(t-s) \otimes \phi(t-s))\left(I_{n} \otimes F(s)\right) S_{[\hat{U}(s)]^{\alpha_{2}}}^{1} d s \\
& \subset(\psi(t) \otimes \phi(t)) \hat{X}_{0}+\int_{0}^{t}(\psi(t-s) \otimes \phi(t-s))\left(I_{n} \otimes F(s)\right) S_{[\hat{U}(s)]^{\alpha_{1}}}^{1} d s .
\end{aligned}
$$

Thus $\hat{X}^{\alpha_{2}} \subset \hat{X}^{\alpha_{1}}$, and hence $[\hat{X}(t)]^{\alpha_{2}} \subset[\hat{X}(t)]^{\alpha_{1}}$. Hence the claim.

Claim (iii). If $\left\{\alpha_{k}\right\}$ is a non-decreasing sequence converging to $\alpha>0$, then $\hat{X}^{\alpha}(t)=\bigcap_{k \geq 1} \hat{X}^{\alpha_{k}}(t)$.

Since $\hat{U}(t)$ is fuzzy set, we have $[\hat{U}(t)]^{\alpha}=\bigcap_{k \geq 1}[\hat{U}(t)]^{\alpha_{k}}$ and then $S_{[\hat{U}(t)]^{\alpha}}^{1}=S_{k \geq 1}^{1}[\hat{U}(t)]^{\alpha_{k}}$. Consider the inclusions

$$
\hat{X}^{\prime}(t) \in G(t) \hat{X}+\left(I_{n} \otimes F(t)\right) \hat{U}^{\alpha_{k}}(t)
$$

and

$$
\hat{X}^{\prime}(t) \in G(t) \hat{X}+\left(I_{n} \otimes F(t)\right) \hat{U}^{\alpha}(t) .
$$

Let $\hat{X}^{\alpha_{k}}$ and $\hat{X}^{\alpha}$ be the solution sets of (3.10) and (3.11) respectively. Therefore

$$
\begin{aligned}
\hat{X}^{\prime}(t) & \in G(t) \hat{X}+\left(I_{n} \otimes F(t)\right)[\hat{U}(t)]^{\alpha} \\
& =G(t) \hat{X}+\left(I_{n} \otimes F(t)\right) \bigcap_{k \geq 1}[\hat{U}(t)]^{\alpha_{k}} \\
& \subset G(t) \hat{X}+\left(I_{n} \otimes F(t)\right)[\hat{U}(t)]^{\alpha_{k}}, \quad \forall(k=1,2, \cdots) .
\end{aligned}
$$


Thus we have $\hat{X}^{\alpha} \subset \hat{X}^{\alpha_{k}}, k=1,2, \cdots$, which implies that

$$
\hat{X}^{\alpha} \subset \bigcap_{k \geq 1} \hat{X}^{\alpha_{k}} .
$$

Let $\hat{X}$ be the solution set to the inclusion

$$
\left[\hat{X}^{\alpha_{k}}(t)\right]^{\prime} \in G(t) \hat{X}^{\alpha_{k}}+\left(I_{n} \otimes F(t)\right)[\hat{U}(t)]^{\alpha_{k}}, k \geq 1 .
$$

Then

$$
\hat{X}(t) \in(\psi(t) \otimes \phi(t)) \hat{X}_{0}+\int_{0}^{t}(\psi(t-s) \otimes \phi(t-s))\left(I_{n} \otimes F(s)\right) S_{[\hat{U}(s)]^{\alpha} \alpha_{k}}^{1} d s,
$$

it follows that

$$
\begin{aligned}
\hat{X}(t) & \in(\psi(t) \otimes \phi(t)) \hat{X}_{0}+\bigcap_{k \geq 1} \int_{0}^{t}(\psi(t-s) \otimes \phi(t-s))\left(I_{n} \otimes F(s)\right) S_{[\hat{U}(s)]^{\alpha_{k}}}^{1} d s \\
& \subset(\psi(t) \otimes \phi(t)) \hat{X}_{0}+\int_{0}^{t}(\psi(t-s) \otimes \phi(t-s))\left(I_{n} \otimes F(s)\right) S_{\bigcap_{k \geq 1}^{1}[\hat{U}(s)]^{\alpha_{k}}} d s \\
& =(\psi(t) \otimes \phi(t)) \hat{X}_{0}+\int_{0}^{t}(\psi(t-s) \otimes \phi(t-s))\left(I_{n} \otimes F(s)\right) S_{[\hat{U}(s)]^{\alpha}}^{1} d s .
\end{aligned}
$$

This implies that $\hat{X} \in \hat{X}^{\alpha}$. Therefore

$$
\bigcap_{k \geq 1} \hat{X}^{\alpha_{k}} \subset \hat{X}^{\alpha}
$$

From (3.12) and (3.13), we have

$$
\hat{X}^{\alpha}=\bigcap_{k \geq 1} \hat{X}^{\alpha_{k}}
$$

and hence

$$
\hat{X}^{\alpha}(t)=\bigcap_{k \geq 1} \hat{X}^{\alpha_{k}}(t)
$$

From Claims (i)-(iii) and applying Theorem 2.1, there exists $\hat{X}(t) \in E^{n^{2}}$ on $[0, T]$ such that $\hat{X}^{\alpha}(t)$ is a solution set to the differential inclusions (3.3) and (3.4). Hence the system (3.1), (3.2) is a fuzzy dynamical Lyapunov system, and it can be expressed as

$$
\begin{gathered}
\hat{X}^{\prime}(t)=G(t) \hat{X}(t)+\left(I_{n} \otimes F(t)\right) \hat{U}(t), \hat{X}(0)=\left\{\hat{X}_{0}\right\} \\
\hat{Y}(t)=\left(I_{n} \otimes C(t)\right) \hat{X}(t)+\left(I_{n} \otimes D(t)\right) \hat{U}(t),
\end{gathered}
$$


The solution set of the fuzzy dynamical system (3.14), (3.15) is given by

$$
\hat{X}(t) \in(\psi(t) \otimes \phi(t)) \hat{X}_{0}+\int_{0}^{t}(\psi(t-s) \otimes \phi(t-s))\left(I_{n} \otimes F(s)\right) \hat{U}(s) d s .
$$

\section{Observability of fuzzy dynamical Lyapunov systems}

In this section we introduce the notion of likely observability and obtain sufficient conditions for fuzzy dynamical system (3.14), (3.15) to be likely observable.

The norm of a matrix $A(t)=\left[a_{i j}(t)\right]$ is defined by $\|A(t)\|=\max _{i j}\left|a_{i j}(t)\right|$ and maximum norm defined by $\|A\|=\max _{t \in[0, T]}\|A(t)\|$.

Definition 4.1. The fuzzy system (3.14), (3.15) is said to be likely observable at $\alpha$-level over the interval $[0, T]$, if the knowledge of the $\alpha$-level input $\hat{U}(t)$ and the $\alpha$-level output $\hat{Y}(t)$ over $[0, T]$ suffices to determine the range of the initial state $\hat{X}_{0}$.

Theorem 4.1. The fuzzy system (3.14), (3.15) is likely observable on level $\alpha$ over the interval $[0, T]$, if $\left(I_{n} \otimes C(T)\right)(\psi(T) \otimes \phi(T))$ is non-singular. Furthermore, let $u_{0}(t)$ and $y_{0}(t)$ be the center points of $\hat{U}(t)$ and $\hat{Y}(t)$ respectively, and let $\widetilde{X}^{\alpha}$ be the possible initial point on $\alpha$-level, then the range estimate for the initial value on $\alpha$-level is given by

$$
\begin{aligned}
\left\|\widetilde{X}^{\alpha}-\hat{X}_{0}\right\| & \leq\left\|\left[\left(I_{n} \otimes C(T)\right)(\psi(T) \otimes \phi(T))\right]^{-1}\right\| \\
& \left(\max _{y_{\alpha}(T) \in \hat{Y}^{\alpha}(T)}\left\|y_{\alpha}(T)-y_{0}(T)\right\|\right. \\
& +\|D(T)\| \max _{u_{\alpha}(T) \in \hat{U}^{\alpha}(T)}\left\|u_{\alpha}(T)-u_{0}(T)\right\| \\
& +\|C(T)\|\|\psi\|\|\phi\|\|F\| \\
& \left.\int_{0}^{T} \max _{u_{\alpha}(t) \in \hat{U}^{\alpha}(t)}\left\|u_{\alpha}(t)-u_{0}(t)\right\| d t\right) .
\end{aligned}
$$

Proof. Let $\hat{X}^{\alpha}$ be the solution set of fuzzy system (3.14), (3.15), then from (3.16), we have

$$
\hat{X}^{\alpha}(T) \in(\psi(T) \otimes \phi(T)) \hat{X}_{0}+\int_{0}^{T}(\psi(T-s) \otimes \phi(T-s))\left(I_{n} \otimes F(s)\right) \hat{U}^{\alpha}(s) d s .
$$


It follows that

$$
\begin{aligned}
\hat{Y}^{\alpha}(T) & \in\left(I_{n} \otimes C(T)\right)\left((\psi(T) \otimes \phi(T)) \hat{X}_{0}\right. \\
& \left.+\int_{0}^{T}(\psi(T-s) \otimes \phi(T-s))\left(I_{n} \otimes F(s)\right) \hat{U}^{\alpha}(s) d s\right)+\left(I_{n} \otimes D(T)\right) \hat{U}^{\alpha}(T),
\end{aligned}
$$

where $\hat{Y}^{\alpha}(T)$ is the $\alpha$-level set of $\hat{Y}(T)$, which implies that

$$
\begin{aligned}
& \left(I_{n} \otimes C(T)\right)(\psi(T) \otimes \phi(T)) \hat{X}_{0} \in \hat{Y}^{\alpha}(T)-\left(I_{n} \otimes D(T)\right) \hat{U}^{\alpha}(T) \\
& \quad-\left(I_{n} \otimes C(T)\right) \int_{0}^{T}(\psi(T-s) \otimes \phi(T-s))\left(I_{n} \otimes F(s)\right) \hat{U}^{\alpha}(s) d s .
\end{aligned}
$$

Let $\widetilde{X}^{\alpha}$ be the possible initial value, then we can write the above equation as

$$
\begin{aligned}
& \left(I_{n} \otimes C(T)\right)(\psi(T) \otimes \phi(T)) \widetilde{X}^{\alpha} \in \hat{Y}^{\alpha}(T)-\left(I_{n} \otimes D(T)\right) \hat{U}^{\alpha}(T) \\
& \quad-\left(I_{n} \otimes C(T)\right) \int_{0}^{T}(\psi(T-s) \otimes \phi(T-s))\left(I_{n} \otimes F(s)\right) \hat{U}^{\alpha}(s) d s .
\end{aligned}
$$

Since $u_{0}, y_{0}$ are the center points of $\hat{U}, \hat{Y}$ respectively, we have

$$
\begin{aligned}
& \left(I_{n} \otimes C(T)\right)(\psi(T) \otimes \phi(T)) \hat{X}_{0}=y_{0}(T)-\left(I_{n} \otimes D(T)\right) u_{0}(T) \\
& \quad-\left(I_{n} \otimes C(T)\right) \int_{0}^{T}(\psi(T-s) \otimes \phi(T-s))\left(I_{n} \otimes F(s)\right) u_{0}(s) d s .
\end{aligned}
$$

From (4.2), (4.3) and the fact that $\left(I_{n} \otimes C(T)\right)(\psi(T) \otimes \phi(T))$ is nonsingular, we can estimate the distance between $\widetilde{X}^{\alpha}$ and $\hat{X}_{0}$ as follows;

$$
\begin{aligned}
& \left\|\left[\left(I_{n} \otimes C(T)\right)(\psi(T) \otimes \phi(T))\right]\left(\widetilde{X}^{\alpha}-\hat{X}_{0}\right)\right\| \\
& \quad \leq \max d\left(\hat{Y}^{\alpha}(T)-\left(I_{n} \otimes D(T)\right) \hat{U}^{\alpha}(T)\right. \\
& \quad-\left(I_{n} \otimes C(T)\right) \int_{0}^{T}(\psi(T-s) \otimes \phi(T-s))\left(I_{n} \otimes F(s)\right) \hat{U}^{\alpha}(s) d s, \\
& \quad y_{0}(T)-\left(I_{n} \otimes D(T)\right) u_{0}(T) \\
& \left.\quad-\left(I_{n} \otimes C(T)\right) \int_{0}^{T}(\psi(T-s) \otimes \phi(T-s))\left(I_{n} \otimes F(s)\right) u_{0}(s) d s\right) \\
& \quad \leq \max _{y_{\alpha}(T) \in \hat{Y}^{\alpha}(T)}\left\|y_{\alpha}(T)-y_{0}(T)\right\|+\|D(T)\| \max _{u_{\alpha}(T) \in \hat{U}^{\alpha}(T)}\left\|u_{\alpha}(T)-u_{0}(T)\right\| \\
& \quad+\|C(T)\|\|\psi\|\|\phi\|\|F\| \int_{0}^{T} \max _{u_{\alpha}(t) \in \hat{U}^{\alpha}(t)}\left\|u_{\alpha}(t)-u_{0}(t)\right\| d t .
\end{aligned}
$$


Hence

$$
\begin{aligned}
\left\|\tilde{X}^{\alpha}-\hat{X}_{0}\right\| \leq \|[( & \left.\left.I_{n} \otimes C(T)\right)(\psi(T) \otimes \phi(T))\right]^{-1} \| \\
& \left(\max _{y_{\alpha}(T) \in \hat{Y}^{\alpha}(T)}\left\|y_{\alpha}(T)-y_{0}(T)\right\|\right. \\
& +\|D(T)\| \max _{u_{\alpha}(T) \in \hat{U}^{\alpha}(T)}\left\|u_{\alpha}(T)-u_{0}(T)\right\| \\
& +\|C(T)\|\|\psi\|\|\phi\|\|F\| \\
& \left.\int_{0}^{T} \max _{u_{\alpha}(t) \in \hat{U}^{\alpha}(t)}\left\|u_{\alpha}(t)-u_{0}(t)\right\| d t\right) .
\end{aligned}
$$

Remark 4.1. The condition of the Theorem 4.1 is only sufficient but not necessary since one level is not enough to determine the non-singularity of $\left(I_{n} \otimes C(T)\right)(\psi(T) \otimes$ $\phi(T))$.

Example 4.1. Consider the fuzzy dynamical matrix Lyapunov system (1.1) satisfying (1.2) with

$$
\begin{gathered}
A(t)=\left[\begin{array}{cc}
0 & -1 \\
1 & 0
\end{array}\right], B(t)=\left[\begin{array}{ll}
1 & 0 \\
0 & 1
\end{array}\right], \quad F(t)=\left[\begin{array}{cc}
e^{t} & 0 \\
0 & e^{t}
\end{array}\right], \\
C(t)=\left[\begin{array}{ll}
0 & 1 \\
1 & 0
\end{array}\right], D(t)=\left[\begin{array}{ll}
0 & 0 \\
0 & 0
\end{array}\right], \text { and } X_{0}=\left[\begin{array}{ll}
1 & 1 \\
1 & 1
\end{array}\right] .
\end{gathered}
$$

Also assume that $\alpha$-level sets of input $\hat{U}(t)$ and output $\hat{Y}(t)$ are

$$
\hat{U}^{\alpha}=\left[\begin{array}{c}
{[\alpha-1,1-\alpha]} \\
{[\alpha-1,1-\alpha]} \\
{[0.1(\alpha-1), 0.1(1-\alpha)]} \\
{[0.1(\alpha-1), 0.1(1-\alpha)]}
\end{array}\right], \quad \hat{Y}^{\alpha}=\left[\begin{array}{c}
{[-\sqrt{1-\alpha}, \sqrt{1-\alpha}]} \\
{[-\sqrt{1-\alpha}, \sqrt{1-\alpha}]} \\
{\left[\frac{1}{2}-\sqrt{1-\alpha}, \frac{1}{2}+\sqrt{1-\alpha}\right]} \\
{\left[\frac{1}{2}-\sqrt{1-\alpha}, \frac{1}{2}+\sqrt{1-\alpha}\right]}
\end{array}\right] .
$$

Then the fundamental matrices of (2.5), (2.6) are

$$
\phi(t)=\left[\begin{array}{cc}
\cos t & -\sin t \\
\sin t & \cos t
\end{array}\right], \psi(t)=\left[\begin{array}{cc}
e^{t} & 0 \\
0 & e^{t}
\end{array}\right] .
$$

Clearly the center points of $\hat{U}(t)$ and $\hat{Y}(t)$ are

$$
u_{0}=[0,0,0,0]^{*}, y_{0}=\left[0,0, \frac{1}{2}, \frac{1}{2}\right]^{*} .
$$

And also

$$
\|\phi\|=\max _{0 \leq t \leq T}\|\phi(t)\|=1, \quad\|\psi\|=\max _{0 \leq t \leq T}\|\psi(t)\|=e^{T},\left\|y_{\alpha}-y_{0}\right\|=\sqrt{1-\alpha},
$$




$$
\begin{gathered}
\left\|\left[\left(I_{n} \otimes C(T)\right)(\psi(T) \otimes \phi(T))\right]^{-1}\right\|=e^{-T},\left\|u_{\alpha}-u_{0}\right\|=1-\alpha, \\
\|F\|=e^{T},\|C\|=1,\|D\|=0 .
\end{gathered}
$$

Substituting these values in (4.1), we have

$$
\left\|\tilde{X}^{\alpha}-\hat{X}_{0}\right\| \leq e^{-T} \sqrt{1-\alpha}+T(1-\alpha) e^{T} .
$$

From the above inequality, it is easily observed that as $T$ becomes larger, our estimated area becomes larger. This means that as $T$ increases it is difficult to determine the initial value. As $\alpha \rightarrow 1$, then $\left\|\widetilde{X}^{\alpha}-\hat{X}_{0}\right\| \rightarrow 0$, i.e., $\widetilde{X}^{\alpha}$ approaches the initial value $\hat{X}_{0}$.

\section{References}

[1] R. J. Aumann, Integrals of set-valued functions, Journal of Mathematical Analysis and Applications, 12(1965), 1-12.

[2] S. Barnett and R. G. Cameron, Introduction to mathematical control theory, Second Edition, Clarendon Press, Oxford (1985).

[3] J. B. Conway, A course in functional analysis, Springer-Verlag, New York, 1990.

[4] G. Debreu, Integration of correspondence, in: Proc. Fifth Berkeley Symp. Math. Statist. Probab., 2, Part 1(Univ. California Press, Berkeley, CA, 1967), 351-372.

[5] Z. Ding and A. Kandel, On the observability of fuzzy dynamical control systems (I), Fuzzy sets and systems, 111(2000), 225-236.

[6] O. Kaleva, Fuzzy differential equations, Fuzzy sets and Systems, 24(1987), 301-317.

[7] V. Lakshmikantham and R. Mohapatra, Theory of Fuzzy Differential Equations and Inclusions, Taylor and Francis, London(2003).

[8] M. S. N. Murty, B. V. Appa Rao and G. Suresh Kumar, Controllability, Observability, and Realizability of matrix Lyapunov systems, Bulletin of the Korean Mathematical Society, 43(1)(2006), 149-159.

[9] M. S. N. Murty and G. Suresh Kumar, Three point boundary value problems for Fuzzy differential equations, Journal of the ChungCheong Mathematical Society, 19(1)(2006), 101-110.

[10] C. V. Negoita and D. A. Ralescu, Applications of fuzzy sets to systems analysis, Willey, New York (1975).

[11] M. L. Puri and D. A. Ralescu, Fuzzy random variables, Journal of Mathematical Analysis and Applications, 114(1986), 409-422.

[12] H. Radstrom, An embedding theorem for space of convex sets, Proc. Amer. Math. Soc. 3(1952), 165-169. 